\title{
Measurement of the tau lepton polarisation at LEP2
}

\section{DELPHI Collaboration}

J. Abdallah ${ }^{\mathrm{z}}$, P. Abreu ${ }^{\mathrm{w}}$, W. Adam ${ }^{\text {bc }}$, P. Adzic ${ }^{1}$, T. Albrecht ${ }^{\mathrm{r}}$, R. Alemany-Fernandez ${ }^{\mathrm{i}}$, T. Allmendinger ${ }^{\mathrm{r}}$, P.P. Allport ${ }^{\mathrm{x}}$, U. Amaldi ${ }^{\text {ad }}$, N. Amapane ${ }^{\mathrm{av}}$, S. Amato ${ }^{\mathrm{az}}$, E. Anashkin ${ }^{\mathrm{ak}}$,

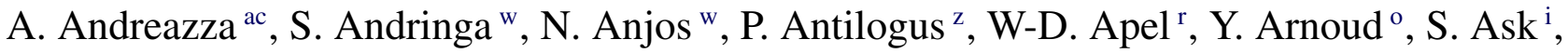
B. Asman ${ }^{\text {au }}$, J.E. Augustin ${ }^{\text {z }}$, A. Augustinus ${ }^{\text {i }}$, P. Baillon ${ }^{\mathrm{i}}$, A. Ballestrero aw, P. Bambade ${ }^{\mathrm{u}}$, R. Barbier ${ }^{\text {ab }}$, D. Bardin ${ }^{\text {q }}$, G.J. Barker ${ }^{\text {be }}$, A. Baroncelli ${ }^{\text {an }}$, M. Battaglia ${ }^{\mathrm{i}}$, M. Baubillier ${ }^{\mathrm{z}}$, K-H. Becks ${ }^{\text {bf }}$, M. Begalli ${ }^{g}$, A. Behrmann ${ }^{\text {bf }}$, E. Ben-Haim ${ }^{\text {u }}$, N. Benekos ${ }^{\text {ag }}$, A. Benvenuti ${ }^{\text {e }}$,

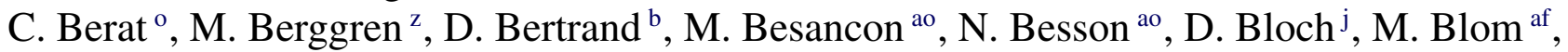
M. Bluj ${ }^{\text {bd }}$, M. Bonesini ${ }^{\text {ad }}$, M. Boonekamp ${ }^{\text {ao }}$, P.S.L. Booth ${ }^{\mathrm{x}, \mathrm{w}}$, G. Borisov ${ }^{\mathrm{v}}$, O. Botner ${ }^{\text {ba }}$, B. Bouquet ${ }^{\text {u }}$, T.J.V. Bowcock ${ }^{\mathrm{x}}$, I. Boyko ${ }^{\mathrm{q}}$, M. Bracko ${ }^{\text {ar }}$, R. Brenner ${ }^{\text {ba }}$, E. Brodet ${ }^{\text {aj, }}$ P. Bruckman ${ }^{\mathrm{s}}$, J.M. Brunet ${ }^{\text {h}}$, B. Buschbeck ${ }^{\text {bc }}$, P. Buschmann ${ }^{\text {bf }}$, M. Calvi ${ }^{\text {ad }}$, T. Camporesi ${ }^{i}$, V. Canale ${ }^{\text {am }}$,

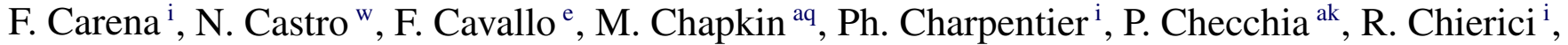
P. Chliapnikov ${ }^{\text {aq }}$, J. Chudoba ${ }^{\mathrm{i}}$, S.U. Chung ${ }^{\mathrm{i}}$, K. Cieslik ${ }^{\mathrm{s}}$, P. Collins ${ }^{\mathrm{i}}$, R. Contri ${ }^{\mathrm{n}}$, G. Cosme ${ }^{\mathrm{u}}$, F. Cossutti ${ }^{\text {ax }}$, M.J. Costa ${ }^{\text {bb }}$, D. Crennell ${ }^{\text {al }}$, J. Cuevas ${ }^{\text {ai }}$, J. D'Hondt ${ }^{\text {b }}$, T. da Silva ${ }^{\text {az }}$, W. Da Silva ${ }^{z}$, D. Dedovich ${ }^{\mathrm{q}}$, G. Della Ricca ${ }^{\text {ax }}$, A. De Angelis ${ }^{\text {ay }}$, W. De Boer ${ }^{\mathrm{r}}$, C. De Clercq ${ }^{\mathrm{b}}$, B. De Lotto ${ }^{\text {ay }}$,

N. De Maria ${ }^{\text {av }}$, A. De Min ${ }^{\text {ak }}$, L. de Paula ${ }^{\text {az }}$, L. Di Ciaccio ${ }^{\text {am }}$, A. Di Simone ${ }^{\text {an }}$, K. Doroba ${ }^{\text {bd }}$, J. Drees ${ }^{\text {bf,i, }}$, G. Eigen ${ }^{\text {d }}$, T. Ekelof ${ }^{\text {ba }}$, M. Ellert ${ }^{\text {ba }}$, M. Elsing ${ }^{\text {i }}$, M.C. Espirito Santo ${ }^{\text {w }}$, G. Fanourakis ${ }^{1}$, D. Fassouliotis ${ }^{1, \mathrm{c}}$, M. Feindt ${ }^{\mathrm{r}}$, J. Fernandez ${ }^{\mathrm{ap}}$, A. Ferrer ${ }^{\mathrm{bb}}$, F. Ferro ${ }^{\mathrm{n}}$, U. Flagmeyer ${ }^{\text {bf }}$, H. Foeth $^{\mathrm{i}}$,

E. Fokitis ${ }^{\text {ag }}$, F. Fulda-Quenzer ${ }^{u}$, J. Fuster ${ }^{\text {bb }}$, M. Gandelman ${ }^{\text {az }}$, C. Garcia ${ }^{\text {bb }}$, Ph. Gavillet ${ }^{\mathrm{i}}$,

E. Gazis ${ }^{\text {ag }}$, R. Gokieli ${ }^{\text {i,bd }}$, B. Golob ${ }^{\text {ar, at }}$, G. Gomez-Ceballos ${ }^{\text {ap }}$, P. Goncalves ${ }^{\text {w }}$, E. Graziani an, G. Grosdidier ${ }^{\text {u }}$, K. Grzelak ${ }^{\text {bd }}$, J. Guy ${ }^{\text {al }}$, C. Haag ${ }^{\mathrm{r}}$, A. Hallgren ${ }^{\text {ba }}$, K. Hamacher ${ }^{\text {bf }}$, K. Hamilton ${ }^{\text {aj }}$, S. Haug ah , F. Hauler ${ }^{\mathrm{r}}$, V. Hedberg aa ${ }^{\text {a }}$ M. Hennecke ${ }^{\mathrm{r}}$, H. Herr ${ }^{\mathrm{i}, \text { }}$, J. Hoffman ${ }^{\text {bd }}$, S-O. Holmgren ${ }^{\text {au }}$, P.J. Holt ${ }^{\mathrm{i}}$, M.A. Houlden ${ }^{\mathrm{x}}$, J.N. Jackson ${ }^{\mathrm{x}}$, G. Jarlskog ${ }^{\text {aa }}$, P. Jarry ${ }^{\text {ao }}$, D. Jeans ${ }^{\text {aj }}$, E.K. Johansson ${ }^{\text {au }}$, P. Jonsson ${ }^{\text {ab }}$, C. Joram ${ }^{\mathrm{i}}$, L. Jungermann ${ }^{\mathrm{r}}$, F. Kapusta ${ }^{\mathrm{z}}$, S. Katsanevas ${ }^{\mathrm{ab}}$, E. Katsoufis ${ }^{\mathrm{ag}}$, G. Kernel ${ }^{\text {ar }}$, B.P. Kersevan ${ }^{\text {ar,at }}$, U. Kerzel ${ }^{\text {r }}$, B.T. King ${ }^{\mathrm{x}}$, N.J. Kjaer ${ }^{\mathrm{i}}$, P. Kluit ${ }^{\text {af }}$, P. Kokkinias ${ }^{1}$, C. Kourkoumelis ${ }^{\mathrm{c}}$,

O. Kouznetsov ${ }^{q}$, Z. Krumstein ${ }^{q}$, M. Kucharczyk ${ }^{\text {s }}$, J. Lamsa ${ }^{\text {a }}$, G. Leder ${ }^{\text {bc }}$, F. Ledroit ${ }^{\circ}$,

L. Leinonen ${ }^{\text {au }}$, R. Leitner ${ }^{\text {ae }}$, J. Lemonne ${ }^{\text {b }}$, V. Lepeltier ${ }^{\text {u }}$, T. Lesiak ${ }^{\text {s }}$, W. Liebig ${ }^{\text {bf }}$, D. Liko ${ }^{\text {bc }}$, A. Lipniacka ${ }^{\text {au }}$, J.H. Lopes ${ }^{\text {az }}$, J.M. Lopez ${ }^{\text {ai }}$, D. Loukas ${ }^{1}$, P. Lutz ${ }^{\text {ao }}$, L. Lyons ${ }^{\text {aj }}$, J. MacNaughton ${ }^{\text {bc }}$,

A. Malek ${ }^{\text {bf }}$, S. Maltezos ${ }^{\text {ag }}$, F. Mandl ${ }^{\text {bc }}$, J. Marco ${ }^{\text {ap }}$, R. Marco ${ }^{\text {ap }}$, B. Marechal ${ }^{\text {az }}$, M. Margonia ${ }^{\text {ak }}$, J-C. Marin ${ }^{\mathrm{i}}$, C. Mariotti ${ }^{\mathrm{i}}$, A. Markou ${ }^{1}$, C. Martinez-Rivero ${ }^{\text {ap }}$, J. Masik ${ }^{\mathrm{m}}$, N. Mastroyiannopoulos ${ }^{1}$,

F. Matorras ap, C. Matteuzzi ${ }^{\text {ad }}$, F. Mazzucato ${ }^{\text {ak }}$, M. Mazzucato ${ }^{\text {ak }}$, R. Mc Nulty ${ }^{\mathrm{x}}$, C. Meroni ${ }^{\mathrm{ac}}$, E. Migliore $^{\text {av }}$, W. Mitaroff ${ }^{\text {bc }}$, U. Mjoernmark ${ }^{\text {aa }}$, T. Moa ${ }^{\text {au }}$, M. Moch ${ }^{\mathrm{r}}$, K. Moenig ${ }^{\mathrm{i}, \mathrm{k}}$, R. Monge $^{\mathrm{n}}$, J. Montenegro $^{\text {af }}$, D. Moraes ${ }^{\text {az }}$, S. Moreno ${ }^{\text {w }}$, P. Morettini ${ }^{\text {n}}$, U. Mueller ${ }^{\text {bf }}$, K. Muenich ${ }^{\text {bf }}$, M. Mulders af ${ }^{\text {, }}$ L. Mundim ${ }^{\mathrm{g}}$, W. Murray ${ }^{\text {al }}$, B. Muryn ${ }^{\mathrm{t}}$, G. Myatt ${ }^{\text {aj }}$, T. Myklebust ${ }^{\text {ah }}$, M. Nassiakou ${ }^{1}$, F. Navarria ${ }^{\mathrm{e}}, \mathrm{K}$. Nawrocki ${ }^{\text {bd }}$, R. Nicolaidou ${ }^{\mathrm{ao}}$, M. Nikolenko ${ }^{\mathrm{q}, \mathrm{j}}$, A. Oblakowska-Mucha ${ }^{\mathrm{t}}$, V. Obraztsov ${ }^{\mathrm{aq}}$, A. Olshevski ${ }^{\mathrm{q}}$, A. Onofre ${ }^{\mathrm{w}}$, R. Orava ${ }^{\mathrm{ar}, \mathrm{p}}, \mathrm{K}$. Osterberg ${ }^{\mathrm{p}}$, A. Ouraou ${ }^{\text {ao }}$, 
A. Oyanguren ${ }^{\text {bb }}$, M. Paganoni ${ }^{\text {ad }}$, S. Paiano ${ }^{\text {e }}$, J.P. Palacios ${ }^{\mathrm{x}}$, H. Palka $^{\text {s }}$, Th.D. Papadopoulou ${ }^{\text {ag }}$, L. Pape $^{\mathrm{i}}$, C. Parkes ${ }^{\mathrm{y}}$, F. Parodi ${ }^{\mathrm{n}}$, U. Parzefall ${ }^{\mathrm{i}}$, A. Passeri ${ }^{\text {an }}$, O. Passon ${ }^{\text {bf }}$, L. Peralta $^{\mathrm{w}}$, V. Perepelitsa ${ }^{\text {bb }}$, A. Perrotta ${ }^{\mathrm{e}}$, A. Petrolini ${ }^{\mathrm{n}}$, J. Piedra ${ }^{\mathrm{a}}$, L. Pieri ${ }^{\text {an }}$, F. Pierre $^{\text {ao }}$, M. Pimenta ${ }^{\mathrm{w}}$, E. Piotto $^{\text {i }}$, T. Podobnik ${ }^{\text {ar, at }}$, V. Poireau ${ }^{\text {i }}$, M.E. Pol ${ }^{\mathrm{f}}$, G. Polok ${ }^{\mathrm{s}}$, V. Pozdniakov ${ }^{\mathrm{q}}$, N. Pukhaeva ${ }^{\mathrm{q}}$, A. Pullia ${ }^{\text {ad }}$, J. Rames ${ }^{\mathrm{m}}$, A. Read ${ }^{\text {ah }}$, P. Rebecchi ${ }^{\mathrm{i}}$, J. Rehn ${ }^{\mathrm{r}}$, D. Reid ${ }^{\text {af }}$, R. Reinhardt ${ }^{\text {bf }}$, P. Renton ${ }^{\text {aj }}$, F. Richard ${ }^{\text {u }}$, J. Ridky ${ }^{\text {m }}$, M. Rivero ${ }^{\text {ap }}$, D. Rodriguez ${ }^{\text {ap }}$, A. Romero ${ }^{\text {av }}$, P. Ronchese ${ }^{\text {ak }}$, P. Roudeau ${ }^{\text {u, }}$ T. Rovelli e , V. Ruhlmann-Kleider ${ }^{\text {ao }}$, D. Ryabtchikov ${ }^{\text {aq }}$, A. Sadovsky ${ }^{\text {q }}$, L. Salmi ${ }^{\text {p }}$, J. Salt ${ }^{\text {bb }}$, C. Sander ${ }^{\text {r }}$ A. Savoy-Navarro ${ }^{\text {z }}$, U. Schwickerath ${ }^{\mathrm{i}}$, R. Sekulin ${ }^{\text {al }}$, M. Siebel ${ }^{\text {bf }}$, A. Sisakian ${ }^{\mathrm{q}}$, G. Smadja ${ }^{\text {ab }}$, O. Smirnova ${ }^{\text {aa }}$, A. Sokolov ${ }^{\text {aq }}$, A. Sopczak ${ }^{\text {, }}$ R. Sosnowski ${ }^{\text {bd }}$, T. Spassov ${ }^{i}$, M. Stanitzki ${ }^{\text {r }}$, A. Stocchi ${ }^{u}$, J. Strauss ${ }^{\text {bc }}$, B. Stugu ${ }^{\text {d }}$, M. Szczekowski ${ }^{\text {bd }}$, M. Szeptycka ${ }^{\text {bd }}$, T. Szumlak ${ }^{t}$, T. Tabarelli ${ }^{\text {ad }}$, F. Tegenfeldt ${ }^{\text {ba }}$, J. Timmermans $^{\text {af, } *}$, L. Tkatchev ${ }^{\mathrm{q}}$, M. Tobin ${ }^{\mathrm{x}}$,

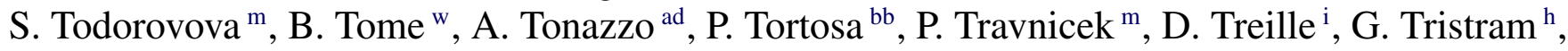
M. Trochimczuk ${ }^{\text {bd }}$, C. Troncon ${ }^{\text {ac }}$, M-L. Turluer ${ }^{\text {ao }}$, I.A. Tyapkin ${ }^{\mathrm{q}}$, P. Tyapkin ${ }^{\mathrm{q}}, \mathrm{S}$. Tzamarias ${ }^{1}$, V. Uvarov ${ }^{\text {aq }}$, G. Valenti ${ }^{\text {e }}$, P. Van Dam ${ }^{\text {af }}$, J. Van Eldik ${ }^{i}$, N. van Remortel ${ }^{p}$, I. Van Vulpen ${ }^{\mathrm{i}}$, G. Vegni ${ }^{\text {ac }}$, F. Veloso ${ }^{w}$, W. Venus ${ }^{\text {al }}$, P. Verdier ${ }^{\text {ab }}$, V. Verzi ${ }^{\text {am }}$, D. Vilanova ${ }^{\text {ao }}$, L. Vitale ${ }^{\text {ax }}$, V. Vrba ${ }^{\mathrm{m}}$, H. Wahlen ${ }^{\text {bf }}$, A.J. Washbrook ${ }^{\mathrm{x}}$, C. Weiser ${ }^{\mathrm{r}}$, D. Wicke ${ }^{\mathrm{i}}$, J. Wickens ${ }^{\mathrm{b}}$, G. Wilkinson ${ }^{\text {aj }}$, M. Winter ${ }^{\mathrm{j}}$, M. Witek ${ }^{\text {s }}$, O. Yushchenko ${ }^{\text {aq }}$, A. Zalewska ${ }^{\text {s }}$, P. Zalewski ${ }^{\text {bd }}$, D. Zavrtanik ${ }^{\text {as }}$, V. Zhuravlov ${ }^{\mathrm{q}}$, N.I. Zimin ${ }^{\mathrm{q}}$, A. Zintchenko ${ }^{\mathrm{q}}$, M. Zupan ${ }^{1}$

\footnotetext{
a Department of Physics and Astronomy, Iowa State University, Ames, IA 50011-3160, USA

b IIHE, ULB-VUB, Pleinlaan 2, B-1050 Brussels, Belgium

${ }^{c}$ Physics Laboratory, University of Athens, Solonos Str. 104, GR-10680 Athens, Greece

d Department of Physics, University of Bergen, Allégaten 55, NO-5007 Bergen, Norway

e Dipartimento di Fisica, Università di Bologna and INFN, Via Irnerio 46, IT-40126 Bologna, Italy

${ }^{\mathrm{f}}$ Centro Brasileiro de Pesquisas Físicas, rua Xavier Sigaud 150, BR-22290 Rio de Janeiro, Brazil

$\mathrm{g}$ Institute de Física, Universidad Estadual do Rio de Janeiro, rua São Francisco Xavier 524, Rio de Janeiro, Brazil

${ }^{\mathrm{h}}$ Collège de France, Laboratoire de Physique Corpusculaire, IN2P3-CNRS, FR-75231 Paris cedex 05, France

${ }^{\mathrm{i}}$ CERN, CH-1211 Geneva 23, Switzerland

${ }^{\mathrm{j}}$ Institut de Recherches Subatomiques, IN2P3-CNRS/ULP-BP20, FR-67037 Strasbourg cedex, France

${ }^{\mathrm{k}}$ Now at DESY-Zeuthen, Platanenallee 6, D-15735 Zeuthen, Germany

${ }^{1}$ Institute of Nuclear Physics, NCSR Demokritos, PO Box 60228, GR-15310 Athens, Greece

${ }^{\mathrm{m}}$ FZU, Institute of Physics of the CAS High Energy Physics Division, Na Slovance 2, CZ-182 21, Praha 8, Czech Republic

${ }^{\mathrm{n}}$ Dipartimento di Fisica, Università di Genova and INFN, Via Dodecaneso 33, IT-16146 Genova, Italy

${ }^{\circ}$ Institut des Sciences Nucléaires, IN2P3-CNRS, Université de Grenoble 1, FR-38026 Grenoble cedex, France

$\mathrm{p}^{\mathrm{p}}$ Helsinki Institute of Physics and Department of Physical Sciences, PO Box 64, FIN-00014 University of Helsinki, Finland

q Joint Institute for Nuclear Research, Dubna, Head Post Office, PO Box 79, RU-101 000 Moscow, Russian Federation

${ }^{\mathrm{r}}$ Institut für Experimentelle Kernphysik, Universität Karlsruhe, Postfach 6980, DE-76128 Karlsruhe, Germany

s Institute of Nuclear Physics PAN, Ul. Radzikowskiego 152, PL-31142 Krakow, Poland

${ }^{\mathrm{t}}$ Faculty of Physics and Nuclear Techniques, University of Mining and Metallurgy, PL-30055 Krakow, Poland

" Université de Paris-Sud, Laboratoire de l'Accélérateur Linéaire, IN2P3-CNRS, Bât. 200, FR-91405 Orsay cedex, France

${ }^{\vee}$ School of Physics and Chemistry, University of Lancaster, Lancaster LA1 4YB, UK

${ }^{\mathrm{w}}$ LIP, IST, FCUL-Av. Elias Garcia, 14-1 ${ }^{\circ}$, PT-1000 Lisboa Codex, Portugal

${ }^{x}$ Department of Physics, University of Liverpool, PO Box 147, Liverpool L69 3BX, UK

${ }^{\mathrm{y}}$ Department of Physics and Astronomy, Kelvin Building, University of Glasgow, Glasgow G12 8QQ, UK

${ }^{\mathrm{z}}$ LPNHE, IN2P3-CNRS, Université Paris VI et VII, Tour 33 (RdC), 4 place Jussieu, FR-75252 Paris cedex 05, France

aa Department of Physics, University of Lund, Sölvegatan 14, SE-223 63 Lund, Sweden

ab Université Claude Bernard de Lyon, IPNL, IN2P3-CNRS, FR-69622 Villeurbanne cedex, France

ac Dipartimento di Fisica, Università di Milano and INFN-MILANO, Via Celoria 16, IT-20133 Milan, Italy

ad Dipartimento di Fisica, Università di Milano-Bicocca and INFN-MILANO, Piazza della Scienza 3, IT-20126 Milan, Italy

ae IPNP of MFF, Charles University, Areal MFF, V Holesovickach 2, CZ-180 00 Praha 8, Czech Republic

af NIKHEF, Postbus 41882, NL-1009 DB Amsterdam, The Netherlands

ag National Technical University, Physics Department, Zografou Campus, GR-15773 Athens, Greece

ah Physics Department, University of Oslo, Blindern, NO-0316 Oslo, Norway

ai Departament de Fisica, Universidad Oviedo, Avda. Calvo Sotelo s/n, ES-33007 Oviedo, Spain

aj Department of Physics, University of Oxford, Keble Road, Oxford OX1 3RH, UK

${ }^{\text {ak }}$ Dipartimento di Fisica, Università di Padova and INFN, Via Marzolo 8, IT-35131 Padua, Italy

${ }^{\text {al }}$ Rutherford Appleton Laboratory, Chilton, Didcot OX11 OQX, UK

am Dipartimento di Fisica, Università di Roma II and INFN, Tor Vergata, IT-00173 Rome, Italy

an Dipartimento di Fisica, Università di Roma III and INFN, Via della Vasca Navale 84, IT-00146 Rome, Italy
} 


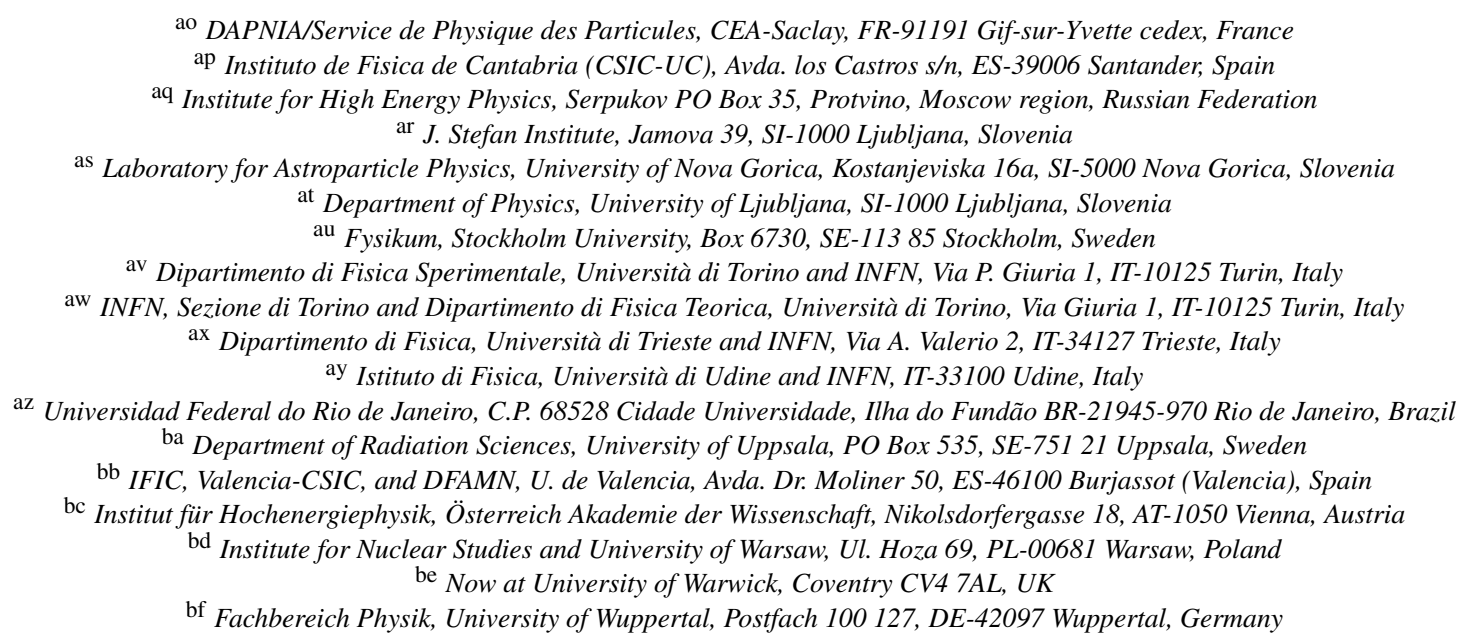

Received 17 April 2007; accepted 1 October 2007

Available online 18 October 2007

Editor: M. Doser

\begin{abstract}
A first measurement of the average polarisation $P_{\tau}$ of tau leptons produced in $\mathrm{e}^{+} \mathrm{e}^{-}$annihilation at energies significantly above the $\mathrm{Z}$ resonance is presented. The polarisation is determined from the kinematic spectra of tau hadronic decays. The measured value $P_{\tau}=-0.164 \pm 0.125$ is consistent with the Standard Model prediction for the mean LEP energy of $197 \mathrm{GeV}$.

(C) 2007 Elsevier B.V. All rights reserved.
\end{abstract}

\section{Introduction}

The polarisation of tau leptons $\left(P_{\tau}\right)$ has been precisely measured by DELPHI [1] and other LEP experiments [2-4] in $\mathrm{Z} \rightarrow \tau^{+} \tau^{-}$decays during the LEP running near the $\mathrm{Z}$ pole (LEP1). The measurements of the tau polarisation allowed the LEP experiments to determine precisely the ratio of the electroweak axial and vector coupling constants, or equivalently, the value of the effective electroweak mixing angle. Starting from 1996 the LEP energy was increased to values significantly above the $\mathrm{Z}$ resonance. In this phase, known as LEP2, the centre-of-mass energy $\sqrt{s}$ of the initial $\mathrm{e}^{+} \mathrm{e}^{-}$system had values lying between 161 and $209 \mathrm{GeV}$. At LEP2, due to the much reduced production cross section, the collected statistics of tau pairs was two orders of magnitude smaller than at LEP1, which makes the experimental errors much larger and therefore they have a weaker constraint on electroweak parameters. However the determination of $P_{\tau}$ at the world's highest energies of $\mathrm{e}^{+} \mathrm{e}^{-}$annihilation is still important for the search for deviations from the Standard Model predictions (e.g. existence of a $Z^{\prime}$ boson).

In this Letter we present the determination of the polarisation of tau leptons produced in $\mathrm{e}^{+} \mathrm{e}^{-}$annihilations at energies between 183 and $209 \mathrm{GeV}$. The data were collected in

\footnotetext{
* Corresponding author.

E-mail address: timmerma@mail.cern.ch (J. Timmermans).

Deceased.
}

the DELPHI experiment during 1997-2000. The data collected during 1996 were not included because of the low integrated luminosity recorded. The analysis was based on the sample of tau pairs selected for the measurement of the production cross section and forward-backward asymmetry [5].

At LEP the tau leptons produced in pairs have opposite helicity. Throughout this Letter we refer to the helicity and polarisation of $\tau^{-}$. The average tau polarisation $P_{\tau}$ is defined as the relative excess of the right-handed $\tau^{-}$over the left-handed ones:

$P_{\tau}=\frac{N_{R}-N_{L}}{N_{R}+N_{L}}$.

The polarisation dependence on the tau production angle was not measured because of too low statistics of the backward tau production at LEP2. In this Letter $P_{\tau}$ denotes the average polarisation over all tau production angles.

At LEP2 a significant fraction of fermion pairs was produced in the radiative return process, when the annihilation energy was reduced to the $\mathrm{Z}$ resonance region by the radiation of a hard photon from the initial state. To ensure that the $\mathrm{e}^{+} \mathrm{e}^{-}$ annihilation occurred at high energy the reconstructed centreof-mass energy of the tau pair $\left(\sqrt{s^{\prime}}\right)$ was required to be close to the nominal LEP energy: $\sqrt{s^{\prime} / s}>0.92$. The determination of $\sqrt{s^{\prime}}$ was based on the measured directions of the jets of tau decay products. The procedure of the tau pair selection and $\sqrt{s^{\prime}}$ determination is described in detail in [5]. The detector calibration and systematic error determination was also largely based 
on the procedures described in [5]. A detailed description of the DELPHI detector and its performance can be found in [6] and [7].

The signal process $\mathrm{e}^{+} \mathrm{e}^{-} \rightarrow \tau^{+} \tau^{-}$was simulated using the KK Monte Carlo generator [8], while tau decays were handled by TAUOLA 2.6 [9]. The main background processes were simulated using the following generators: BHWIDE [10] for $\mathrm{e}^{+} \mathrm{e}^{-} \rightarrow \mathrm{e}^{+} \mathrm{e}^{-} ;$KK for $\mathrm{e}^{+} \mathrm{e}^{-} \rightarrow \mu^{+} \mu^{-} ;$KK and PYTHIA [11] for $\mathrm{e}^{+} \mathrm{e}^{-} \rightarrow q \bar{q}$; WPHACT [12] for $\mathrm{e}^{+} \mathrm{e}^{-} \rightarrow \mathrm{W}^{+} \mathrm{W}^{-}, \mathrm{e}^{+} \mathrm{e}^{-} \rightarrow$ $\mathrm{ZZ}$ and $\mathrm{e}^{+} \mathrm{e}^{-} \rightarrow \mathrm{Ze}^{+} \mathrm{e}^{-}$; BDK/BDKRC [13] for $\gamma \gamma \rightarrow \mathrm{e}^{+} \mathrm{e}^{-}$, $\gamma \gamma \rightarrow \mu^{+} \mu^{-}$and $\gamma \gamma \rightarrow \tau^{+} \tau^{-}$; and PYTHIA for $\gamma \gamma \rightarrow q \bar{q}$. The generated events were passed through the full chain of the detector simulation, event reconstruction and data analysis. The procedure of the Monte Carlo simulation of the DELPHI detector is described in [7].

\section{Event selection}

The determination of the average tau polarisation was based on the inclusive selection of one-prong hadronic decays of tau leptons. Leptonic and multi-track tau decays were not used because of their very low sensitivity to the polarisation. The method closely followed the one developed for the LEP1 analysis [1], with modifications necessary to take into account the increased centre-of-mass energy and the lower number of tau pairs observed at LEP2. The charged particles in each preselected event were combined into two jets using the PYCLUS algorithm [11]. The most energetic charged particle (leading track) was determined for each jet and all tracks and electromagnetic showers within a $30^{\circ}$ cone around each leading track were assumed to originate from the decay of the tau lepton. The two tau decay candidates in each event were then analysed separately. An important quantity for this analysis, the visible invariant mass ( $M_{\mathrm{VIS}}$ ), was calculated for each tau decay candidate using all charged particles (assumed to be pions) and all photons, i.e. electromagnetic showers with energy above $0.5 \mathrm{GeV}$ unassociated with a charged particle.

The one-prong hadronic tau decays were selected using the following procedure. The leading track had to be reconstructed within the barrel part of the DELPHI detector (polar angle ${ }^{1}$ range $41^{\circ}<\theta<139^{\circ}$ ). Tracks close to the DELPHI middle plane $\left(88.5^{\circ}<\theta<91.5^{\circ}\right)$ were excluded. Tau decay candidates in which the leading track extrapolation passed closer than $0.3^{\circ}$ from the centre of a $\phi$-crack of the barrel electromagnetic calorimeter (HPC) were also excluded. The leading track had to be the only track originating from the tau decay, with the exception of the tracks that were reconstructed as an $\mathrm{e}^{+} \mathrm{e}^{-}$pair from a conversion (such pairs were treated as photons in the analysis). The procedure of the conversion reconstruction is described in [7].

Tau decays to electrons of relatively low energy were rejected by the requirement that the measured $d E / d x$ losses of

\footnotetext{
1 The DELPHI coordinate system is a right-handed system with the $z$-axis collinear with the incoming electron beam, the $x$-axis pointing to the centre of the LEP accelerator and the $y$-axis vertical. The polar angle $\theta$ is with reference to the $z$-axis, and $\phi$ is the azimuthal angle in the $x, y$ plane.
}

the charged particle as measured in the Time Projection Chamber (TPC) did not exceed the value expected for a pion by more than 2 standard deviations. Electrons of higher energies were suppressed by requiring that at least one of the two following conditions was satisfied: either the energy deposition in the HPC associated to the charged particle had to be less than $10 \mathrm{GeV}$ or the associated deposition beyond the first layer of the Hadron Calorimeter (HCAL) had to be greater than $0.5 \mathrm{GeV}$. In the cases where a $d E / d x$ measurement was not available, the event was rejected if the particle momentum was in the range below $10 \mathrm{GeV} / c$ for which the HPC energy measurement is less precise.

The tau decays involving muons were suppressed by the requirement that no hits in the muon chambers were associated to the charged particle by the standard DELPHI procedure of muon identification [7]. For the tau decay candidates with low visible invariant mass $\left(M_{\mathrm{VIS}}<0.3 \mathrm{GeV} / c^{2}\right)$ an additional muon-suppression was applied: the average measured energy deposition per HCAL layer associated to the charged particle had to be inconsistent with a minimum ionizing particle, namely it had to lie outside the range 0.5 to $1.5 \mathrm{GeV}$.

During the whole period of data taking in 2000 the performance of one of the 12 sectors of the DELPHI TPC was unstable. The good performance of the TPC is crucial for this analysis, in particular for the $d E / d x$ measurements. Therefore for the data taken in 2000 the selection procedure was modified. A tau decay candidate was rejected if the leading track was reconstructed within the faulty TPC sector or close to it (within $10^{\circ}$ in azimuthal angle). This reduced the selection efficiency for the 2000 data by approximately $10 \%$.

Two of the event selection variables are illustrated in Fig. 1. The upper plot shows the distribution of the so-called " $d E / d x$ pull" for the pion hypothesis, i.e. the difference between the measured $d E / d x$ losses of the charged particle and the value expected for a pion, expressed in number of standard deviations (see [1] for the exact definition), for particles with momentum below $12 \mathrm{GeV} / c$. The lower plot shows the distribution of the average energy deposition per HCAL layer associated to the charged particle. The grey areas in Fig. 1 show the background most relevant to the variable shown. The data shown in Fig. 1 represent the full statistics of 1997-2000.

In total, 624 hadronic tau decay candidates were selected from the 1997-2000 data. The details of the selection for each year of data taking are summarised in Table 1. The efficiency values are given within the polar angle acceptance. The efficiency drop in 2000 is due to the rejection of particles crossing the faulty TPC sector. The non-tau background consisted mainly of $\mathrm{e}^{+} \mathrm{e}^{-} \rightarrow \mathrm{e}^{+} \mathrm{e}^{-}, \mathrm{e}^{+} \mathrm{e}^{-} \rightarrow \mathrm{W}^{+} \mathrm{W}^{-}$and $\mathrm{e}^{+} \mathrm{e}^{-} \rightarrow$ $\mathrm{Ze}^{+} \mathrm{e}^{-}$events (in approximately equal fractions). The selection efficiency and the background level were determined from the simulation. Small corrections (typically 10\%) were applied to the residual non-tau background to account for the differences between data and simulation. The procedure for this correction is described in [5].

Fig. 2 shows the dependence of the selection efficiency on the variables which are sensitive to the tau polarisation: momentum of the charged particle; total energy of photons from 


\section{$D E L P H I$}
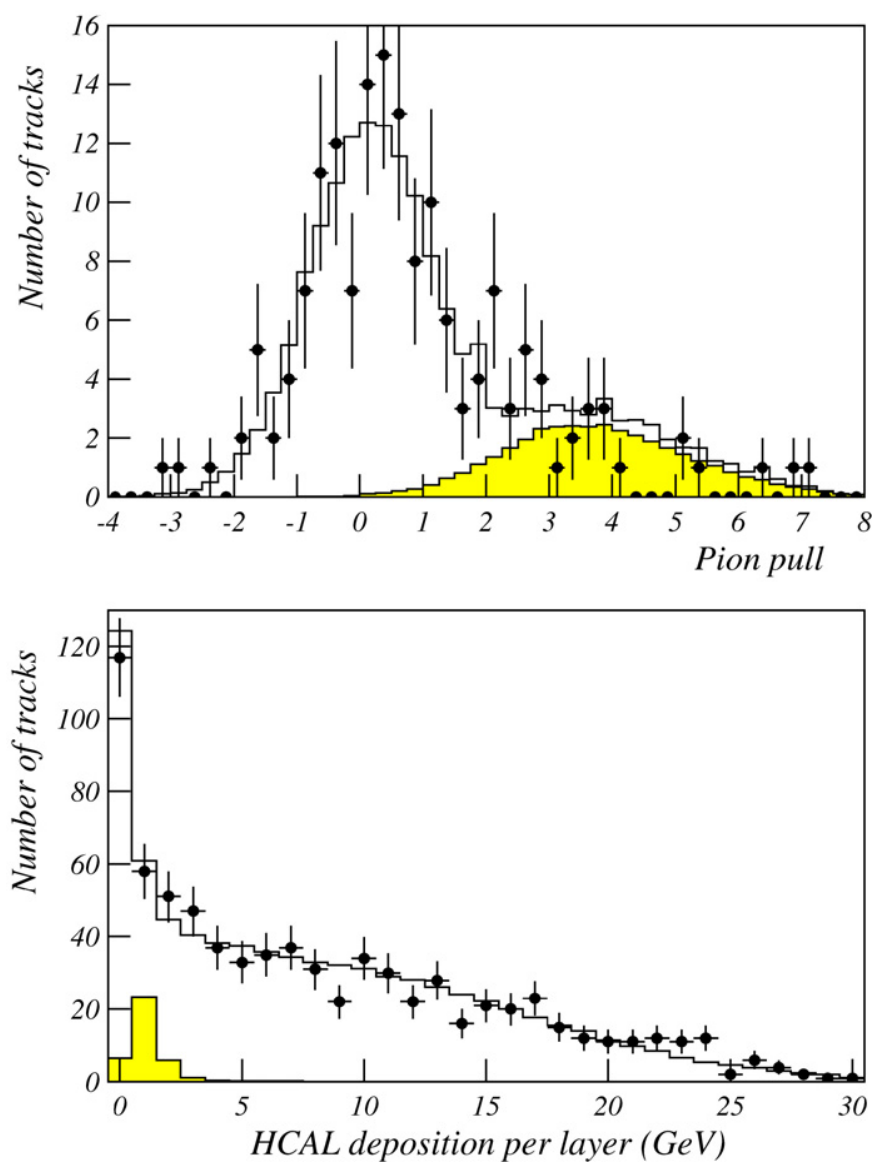

Fig. 1. Top: distribution of the $d E / d x$ pion hypothesis pull. The grey/yellow area shows the contribution expected from electrons. Bottom: distribution of the average energy deposition per HCAL layer. The grey/yellow area shows the contribution from muons. In both plots the real data are represented by points and the solid lines show the simulation. (For interpretation of the references to colour in this figure legend, the reader is referred to the web version of this Letter.)

Table 1

Results of the tau hadronic decay selection

\begin{tabular}{lrrrr}
\hline Year & 1997 & 1998 & 1999 & 2000 \\
\hline Mean $\sqrt{s}(\mathrm{GeV})$ & 183 & 189 & 198 & 206 \\
Integrated luminosity $\left(\mathrm{pb}^{-1}\right)$ & 52 & 153 & 224 & 217 \\
Number of selected tau pairs (in barrel) & 82 & 231 & 305 & 254 \\
Number of selected hadronic tau decays & 56 & 159 & 234 & 175 \\
Hadronic selection efficiency $(\%)$ & 77.3 & 77.1 & 77.1 & 70.3 \\
Non-tau background $(\%)$ & 4.6 & 3.8 & 4.7 & 4.4 \\
Tau leptonic decay background $(\%)$ & 3.3 & 3.4 & 3.2 & 3.3 \\
Fraction $(\%)$ of events with $\sqrt{s^{\prime} / s}<0.92$ & 5.3 & 4.9 & 4.9 & 5.0 \\
\hline
\end{tabular}

the tau decay; and $M_{\mathrm{VIS}}$. The step at $10 \mathrm{GeV} / c$ momentum is caused by the different treatment of the tracks without $d E / d x$ measurement. The drop of efficiency at low invariant masses is due to the tighter muon rejection in this region. In general, the efficiency is relatively flat, which is important for an unbiased polarisation measurement.

The distribution of the visible invariant mass for the selected decays is shown in Fig. 3. The main plot does not show the first
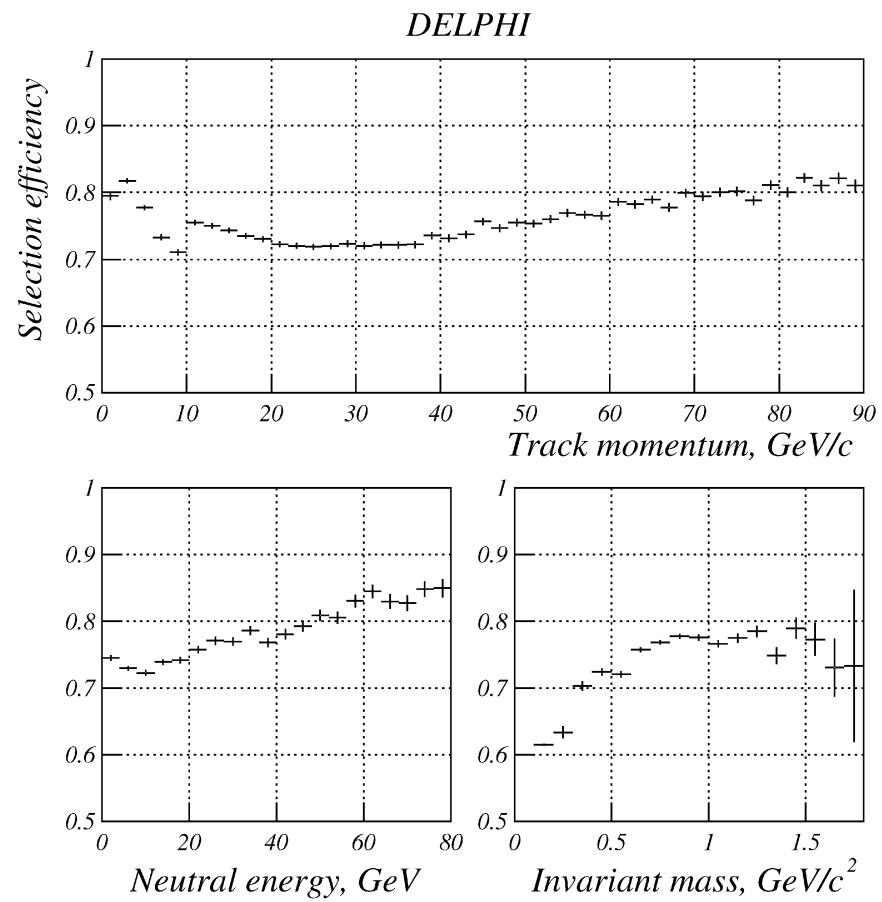

Fig. 2. Efficiency of the 1-prong hadronic tau decay selection versus the kinematic variables: momentum of the charged particle; total energy of photons; and the visible invariant mass of tau decay products. The error bars represent the statistical uncertainty of the simulation sample. The step at $10 \mathrm{GeV} / \mathrm{c}$ (upper plot) is caused by the rejection of tracks without $d E / d x$ measurement.

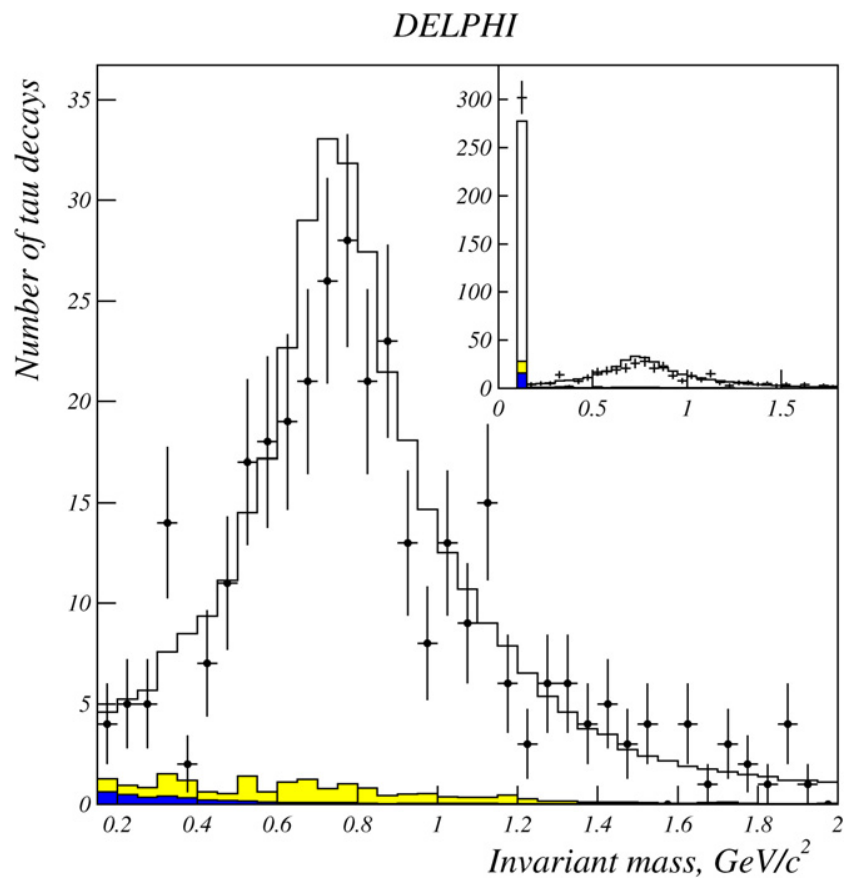

Fig. 3. Distribution of the visible invariant mass. The points represent data, the solid line is the simulation, and the grey/yellow and black/blue areas show the contributions respectively from non-tau background and from leptonic tau decays. The main plot and the inset show the same distributions in different scale. (For interpretation of the references to colour in this figure legend, the reader is referred to the web version of this Letter.)

bin corresponding to $\tau \rightarrow \pi v$ decays. The same distribution, including the first bin, is shown in the inset. 


\section{Determination of the tau polarisation}

The selected sample mainly consisted of the decays $\tau \rightarrow \pi \nu$, $\tau \rightarrow \rho \nu$ and $\tau \rightarrow a_{1} \nu$. Mixing the different decay modes in the inclusive sample reduces the analysis sensitivity to the polarisation. In order to improve the sensitivity the analysis was performed in three bins of the visible invariant mass: $0<M_{\mathrm{VIS}}<0.3 \mathrm{GeV} / c^{2}$, dominated by $\tau \rightarrow \pi \nu(59 \%)$; $0.3 \mathrm{GeV} / c^{2}<M_{\mathrm{VIS}}<0.8 \mathrm{GeV} / c^{2}$, dominated by $\tau \rightarrow \rho \nu$ (78\%); and $0.8 \mathrm{GeV} / c^{2}<M_{\mathrm{VIS}}<2.0 \mathrm{GeV} / c^{2}$, populated by $\tau \rightarrow \rho \nu(61 \%)$ and $\tau \rightarrow a_{1} v(34 \%)$. The total numbers of decays selected in each bin of $M_{\mathrm{VIS}}$ were 316,153 and 155, respectively.

As in the LEP1 analysis [1] the extraction of the tau polarisation was based on reconstruction of the two kinematic variables characterizing the tau decay: $\Theta$, the angle in the $\tau$ rest frame between the momenta of $\tau$ and $h$ for $\tau \rightarrow h \nu$ decays; and $\Psi$ which, in the case of $\tau \rightarrow \rho v$ decay, is the angle of the emission of the pions in the $\rho$ rest frame. The angle $\Theta$ was reconstructed as

$\cos \Theta=\frac{2 p_{h} / p_{\tau}-1-m_{h}^{2} / m_{\tau}^{2}}{1-m_{h}^{2} / m_{\tau}^{2}}$,

where $p_{h}$ is the momentum of the hadronic system produced in the tau decay (vector sum of the momenta of the reconstructed tau decay products) and $m_{h}$ is the mass of the hadronic system (experimentally reconstructed as $M_{\mathrm{VIS}}$ ). The tau lepton momentum $p_{\tau}$ was estimated from the directions of the jets of the tau decay products using the same method as for the determination of the $\sqrt{s^{\prime}}$ value (see [5] for a detailed explanation). The uncertainty of the $p_{\tau}$ determination was approximately $1.5 \%$, mainly due to the unknown energies and directions of the neutrinos produced in the tau decays. The angle $\Psi$ was determined from

$\cos \Psi=\frac{E_{\mathrm{ch}}-E_{\text {neu }}}{E_{\mathrm{ch}}+E_{\text {neu }}}$,

where $E_{\mathrm{ch}}$ and $E_{\text {neu }}$ are the energy of the charged particle and the total energy of the photons from the tau decay. For visible invariant masses above $0.3 \mathrm{GeV} / c^{2}$ the range $\cos \Theta>0.8$ was rejected because it was dominated by events with wrongly reconstructed kinematics.

The value of the tau polarisation was extracted from a binned likehood fit to the observed distributions of $\cos \Theta$ and $\cos \Psi$ by the simulation expectation $f_{\mathrm{MC}}$ with the $P_{\tau}$ value being a free fit parameter:

$f_{\mathrm{MC}}=f_{\mathrm{bg}}+R \cdot\left(\frac{1-P_{\tau}}{1-P_{0}} f_{L}+\frac{1+P_{\tau}}{1+P_{0}} f_{R}\right)$,

where $f_{\mathrm{bg}}, f_{L}$ and $f_{R}$ are the contributions from external (nontau) background and from decays of left- and right-handed tau leptons, and $P_{0}$ is the generator level tau polarisation in the simulated tau pair sample. The external background contribution was normalized to the luminosity. The factor $R$ normalizes the number of events in the simulated tau signal to the real data after external background subtraction:

$R \cdot N_{\mathrm{MC}}^{\tau}=N_{\text {data }}-N_{\mathrm{bg}}$,
Table 2

Values of the tau polarisation determined from each year's data, and their average. Also shown are the statistical errors from the fits and the uncertainty due to the limited statistics of the simulation samples

\begin{tabular}{llll}
\hline Year & $\tau$ polarisation & Stat. error & Simulation stat. error \\
\hline 1997 & -0.61 & 0.34 & 0.015 \\
1998 & -0.41 & 0.21 & 0.009 \\
1999 & -0.01 & 0.20 & 0.009 \\
2000 & +0.11 & 0.24 & 0.010 \\
Average & -0.176 & 0.117 & 0.005 \\
\hline
\end{tabular}

where $N_{\text {data }}$ is the number of observed events, $N_{\text {MC }}^{\tau}$ is the number of simulated signal events, and $N_{\mathrm{bg}}$ is the non-tau background predicted by simulation. Such a fit automatically takes into account the bias due to different selection efficiencies for different tau helicities. It does not depend on the tau polarisation in the simulated tau pair sample.

The tau polarisation was extracted separately for each year of the data taking. The two-dimensional distributions of $\cos \Theta$ versus $\cos \Psi$ were fitted simultaneously in the three bins of the invariant mass. For the first bin of invariant mass only the onedimensional distribution of $\cos \Theta$ was used because this bin is dominated by decays to pions where $\Psi$ has no meaning. The results of the fits are presented in Table 2, together with their average. The Table also shows the statistical uncertainty of the $P_{\tau}$ determination and the uncertainties associated with the finite statistics of the simulated events. This Table shows the results obtained from the fit before applying the corrections discussed in the next section. Despite the apparent energy dependence, the results are consistent with being constant with energy. The $\chi^{2} /$ n.d.f. for a constant value is $5.0 / 3$.

As a cross-check, the result was also obtained with a single fit to the whole data sample (1997-2000). The Monte Carlo samples were combined with weights proportional to the integrated luminosity of the respective year. The result of this fit was $-0.140 \pm 0.123$, which is less than one standard deviation from the average in Table 2 (allowing for the high statistical correlation between both values). The average of the year-by-year measurements was chosen to produce the final result because the year-specific Monte Carlo samples should better reproduce differences in detector performance and calibration in the different periods of data taking.

The results of the fit are illustrated in Fig. 4 which shows the distribution of $\cos \Theta$ for the first bin of invariant mass and one-dimensional projections of the fitted two-dimensional distributions for other invariant masses. Combined data of all years are shown by the points with error bars and the simulation is shown by the solid lines. The distributions for simulated tau decays are shown with the polarisation value which was obtained in this study. The contributions from the decays of left- and right-handed tau leptons are shown by the dashed and dotted lines respectively. The contribution of the non-tau background is shown as a grey/yellow area.

\section{Corrections and systematic errors}

A small correction had to be applied to the measured polarisation to subtract the contribution of the feed-through events, 


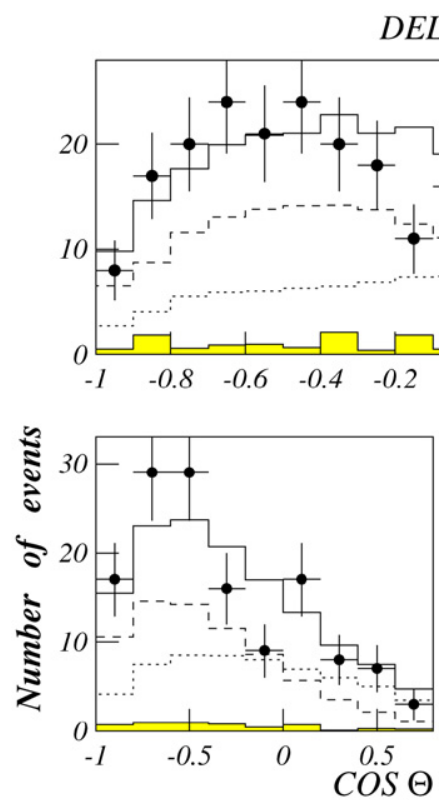

DELPHI
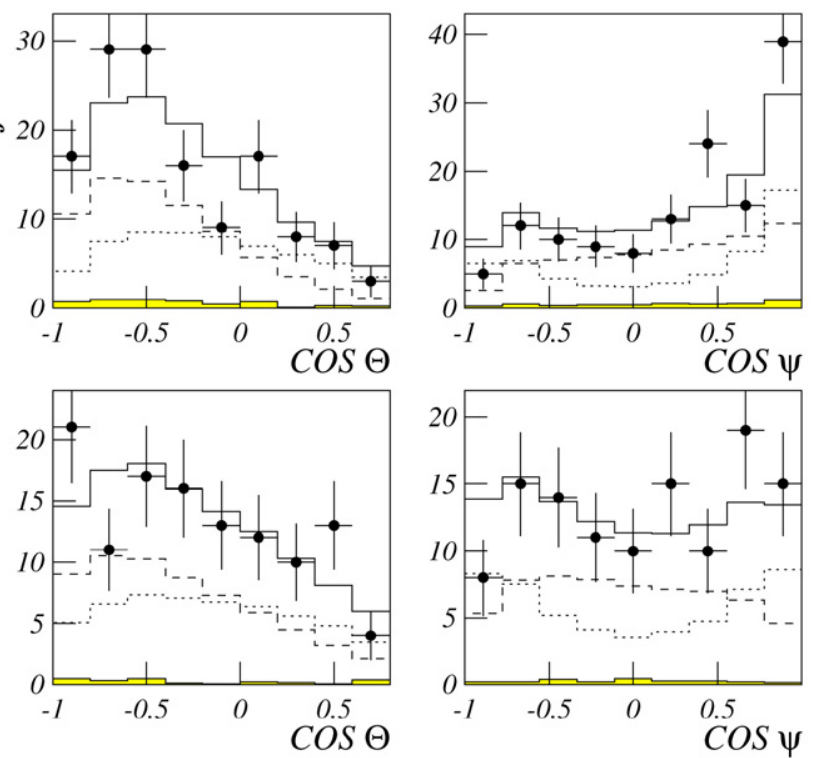

Fig. 4. The results of the tau polarisation fit for different bins of $M_{\text {VIS }}: 0-0.3 \mathrm{GeV} / c^{2}$ (upper plot), $0.3-0.8 \mathrm{GeV} / c^{2}$ (middle plots) and $0.8-2.0 \mathrm{GeV} / c^{2}$ (lower plots). The points represent data, the grey/yellow areas show the non-tau background, the dashed and dotted lines show the contributions from the decays of left- and right-handed tau leptons, and the solid lines show the total prediction of simulation. (For interpretation of the references to colour in this figure legend, the reader is referred to the web version of this Letter.)

i.e. the events which have true values of $\sqrt{s^{\prime} / s}$ below 0.92 although they pass the experimental cut of $\sqrt{s^{\prime} / s}>0.92$ (see Table 1). After such a correction the measured polarisation represents the average polarisation of tau leptons produced at the actual annihilation energies above $0.92 \cdot \sqrt{s}$. The value of the correction depends on the measured polarisation. Since the results from individual years (Table 2) are consistent with each other, and the polarisation dependence on energy is weak, we apply to the results of all years the same global correction calculated using the KK generator for the average measured polarisation. The value of the correction was found to be +0.004 .

This method of tau polarisation measurement depends on a good description of the data by the simulation. Therefore an extensive study of the simulation quality has been performed using high purity test samples selected from data and simulation. The uncertainties of such checks (dominated by the statistics of test samples selected from data) were converted into the systematic uncertainty of the polarisation measurement. To reduce the effect of statistical fluctuations, the test samples were selected from the combined 1997-2000 data. The systematic uncertainties therefore were common to all years of the data taking. Most of the corrections and corresponding systematic uncertainties were propagated from the study of tau pair production, see [5]. Some of these correspond to small corrections applied to variables at the very beginning of the analysis, before the tau pair selection, such as the correction to the measured $d E / d x$ (see below), which are therefore already included in the results of Table 2 . In other cases they had to be calculated as corrections to the results and have to be added to those. In these cases the correction values are given below. A conservative approach was followed, applying a correction and uncertainty even in the cases where the correction was consistent with zero.

The $d E / d x$ measurements were calibrated using test samples of muons from the processes $\gamma \gamma \rightarrow \mu^{+} \mu^{-}, \mathrm{e}^{+} \mathrm{e}^{-} \rightarrow$ $\mu^{+} \mu^{-}$and $\mathrm{Z} \rightarrow \mu^{+} \mu^{-}$(the latter were produced during the short periods of LEP running near the $\mathrm{Z}$ pole in 1997-2000). Both the $d E / d x$ mean value and the measurement resolution were calibrated and a small momentum-dependent correction was applied. The uncertainty due to the calibration gave rise to an uncertainty of \pm 0.017 in $P_{\tau}$.

The measurement of photon energy was important for the reconstruction of the tau hadronic decay kinematics. The electromagnetic energy scale was checked using a sample of electrons from $\gamma \gamma \rightarrow \mathrm{e}^{+} \mathrm{e}^{-}, \mathrm{e}^{+} \mathrm{e}^{-} \rightarrow \mathrm{e}^{+} \mathrm{e}^{-}$and $\mathrm{Z} \rightarrow \mathrm{e}^{+} \mathrm{e}^{-}$events. A correction of $-0.010 \pm 0.010$ to the tau polarisation was found to be necessary.

The redundancy between the HPC and HCAL was used to estimate from the data the efficiency of the "HPC or HCAL" cut which rejects electrons. The momentum dependence of the cut efficiency was found to be slightly different in data and in simulation. A correction of $+0.018 \pm 0.022$ was applied to the $P_{\tau}$ value.

From the data/simulation comparison for the distribution of the number of reconstructed photons in tau hadronic decays it was found that the photon reconstruction efficiency was well described by the simulation. The uncertainty of this check resulted in a \pm 0.016 uncertainty on the $P_{\tau}$ value.

The efficiency of the muon rejection cuts was checked using the redundancy of the HCAL and the muon chambers. The muon chamber efficiency was slightly (4-7\%) higher in simulation than in the data. The discrepancy was corrected by randomly removing a fraction of muon chamber hits in simulation. An uncertainty of \pm 0.012 on $P_{\tau}$ was associated with this correction.

The systematic uncertainty associated with the residual background level was determined by varying the background by $\pm 20 \%$. The size of this variation was estimated from the small residual data/simulation disagreements in the shapes of background-sensitive distributions. The statistical contribution from the number of simulated background events was negligible. The resultant $P_{\tau}$ uncertainty was \pm 0.014 for the background from tau leptonic decays and \pm 0.004 for the non-tau background.

Other possible systematic errors were estimated from variations of the selection cuts and from changing the choice of binning of the variables used in the fit of the tau polarisation. 
The full list of systematic errors is summarized in Table 3. Where necessary the corrections to the measured tau polarisation are also given.

\section{Results and conclusions}

As can be seen in Table 3 the total correction that has to be applied to the observed value of the tau polarisation is +0.012 . After taking into account this correction the average tau lepton polarisation measured at LEP2 is

$P_{\tau}=-0.164 \pm 0.117 \pm 0.045$,

where the first uncertainty is statistical and the second is systematic. Fig. 5 presents the centre-of-mass energy dependence of the tau polarisation measured by the DELPHI experiment. The plot shows the LEP1 precision measurement and the mea-

Table 3

Summary of systematic uncertainties and corrections to the tau polarisation. All values are in units of $10^{-3}$

\begin{tabular}{lcc}
\hline Source & $P_{\tau}$ uncertainty & $P_{\tau}$ correction \\
\hline$d E / d x$ calibration & 17 & - \\
$E_{\gamma}$ scale & 10 & -10 \\
"HPC or HCAL" efficiency & 22 & +18 \\
$\gamma$ reconstr. efficiency & 16 & - \\
Muon chamber efficiency & 12 & - \\
Internal background & 14 & - \\
External background & 4 & - \\
Variation of cuts & 9 & - \\
Binning choice & 20 & - \\
Simulation statistics & 5 & - \\
Feed-through & - & +4 \\
Total & 45 & +12
\end{tabular}

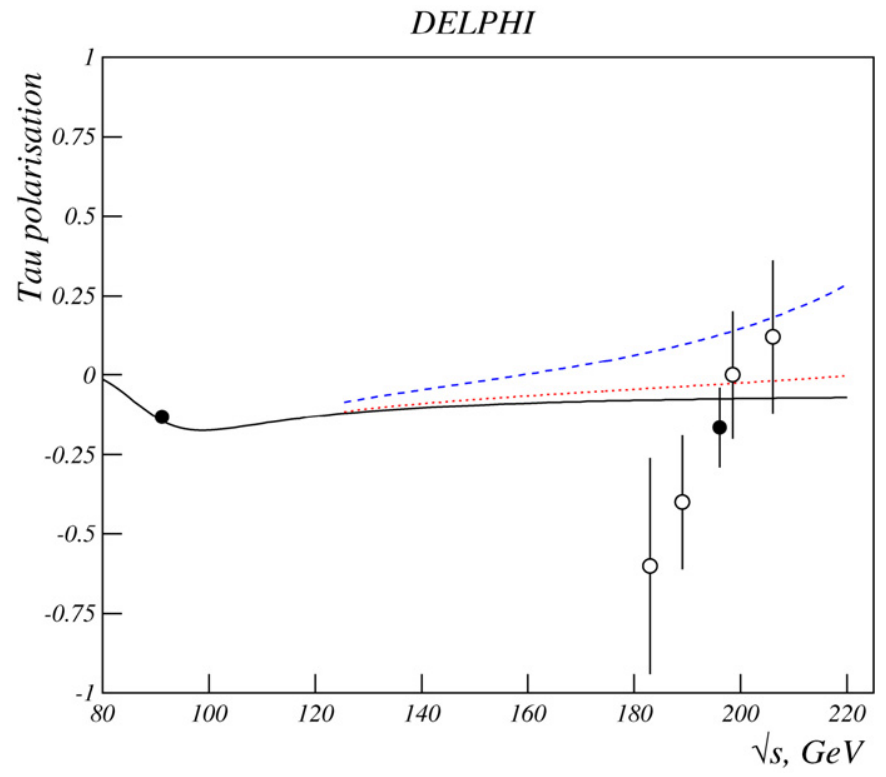

Fig. 5. Energy dependence of the tau polarisation. Black circles show the average DELPHI measurements at LEP1 and LEP2. The white circles are the DELPHI measurements at different LEP2 energies. The solid line shows the Standard Model prediction (ZFITTER 6.36). The dashed and dotted lines show the effects from 300 and $455 \mathrm{GeV} / c^{2} \mathrm{Z}^{\prime}$ bosons, respectively. surements at the four LEP2 energies. Also shown is the average LEP2 value which corresponds to a luminosity-weighted mean collision energy of $197 \mathrm{GeV}$. The solid curve shows the theoretical predictions calculated using the ZFITTER version 6.36 package [14]. The calculations used the Standard Model parameters determined at LEP1 and SLD [15]. Two other curves illustrate the effect of the existence of a $\mathrm{Z}^{\prime}$ boson in left-right models, assuming $\alpha_{\mathrm{LR}}=\sqrt{2 / 3}$ [16]. The dashed curve corresponds to $M_{\mathrm{Z}^{\prime}}=300 \mathrm{GeV} / c^{2}$ and the dotted curve represents the DELPHI limit $M_{\mathrm{Z}^{\prime}}=455 \mathrm{GeV} / c^{2}$ derived from the measured fermion pair production cross section and charge asymmetry [5].

In summary, we have measured the polarisation of tau leptons produced at the world's highest $\mathrm{e}^{+} \mathrm{e}^{-}$annihilation energy. The values measured at different energies between 183 and $209 \mathrm{GeV}$ are consistent. The average tau polarisation value $-0.164 \pm 0.125$ is consistent with the Standard Model prediction of -0.075 at the corresponding mean energy of $197 \mathrm{GeV}$. This measurement excludes positive values of the tau polarisation at the $90 \%$ confidence level.

\section{Acknowledgements}

We are greatly indebted to our technical collaborators, to the members of the CERN-SL Division for the excellent performance of the LEP collider, and to the funding agencies for their support in building and operating the DELPHI detector.

We acknowledge in particular the support of Austrian Federal Ministry of Education, Science and Culture, GZ 616.364/2III/2a/98, FNRS-FWO, Flanders Institute to encourage scientific and technological research in the industry (IWT) and Belgian Federal Office for Scientific, Technical and Cultural affairs (OSTC), Belgium; FINEP, CNPq, CAPES, FUJB and FAPERJ, Brazil; Ministry of Education of the Czech Republic, project LC527; Academy of Sciences of the Czech Republic, project AV0Z10100502; Commission of the European Communities (DG XII); Direction des Sciences de la Matière, CEA, France; Bundesministerium für Bildung, Wissenschaft, Forschung und Technologie, Germany; General Secretariat for Research and Technology, Greece; National Science Foundation (NWO) and Foundation for Research on Matter (FOM), The Netherlands; Norwegian Research Council; State Committee for Scientific Research, Poland, SPUB-M/CERN/PO3/DZ296/2000, SPUBM/CERN/PO3/DZ297/2000, 2P03B 10419 and 2P03B 69 23(2002-2004); FCT, Fundação para a Ciência e Tecnologia, Portugal; Vedecka grantova agentura MS SR, Slovakia, Nr. 95/5195/134; Ministry of Science and Technology of the Republic of Slovenia; CICYT, Spain, AEN99-0950 and AEN990761; The Swedish Research Council, Particle Physics and Astronomy Research Council, UK; Department of Energy, USA, DE-FG02-01ER41155; EEC RTN contract HPRN-CT-002922002.

\section{References}

[1] DELPHI Collaboration, P. Abreu, et al., Eur. Phys. J. C 14 (2000) 585. [2] ALEPH Collaboration, A. Heister, et al., Eur. Phys. J. C 20 (2001) 401. 
[3] L3 Collaboration, M. Acciarri, et al., Phys. Lett. B 429 (1998) 387.

[4] OPAL Collaboration, G. Abbiendi, et al., Eur. Phys. J. C 21 (2001) 1.

[5] DELPHI Collaboration, J. Abdallah, et al., Eur. Phys. J. C 45 (2006) 589.

[6] DELPHI Collaboration, P. Aarnio, et al., Nucl. Instrum. Methods A 303 (1991) 233.

[7] DELPHI Collaboration, P. Abreu, et al., Nucl. Instrum. Methods A 378 (1996) 57.

[8] S. Jadach, B.F.L. Ward, Z. Was, Comput. Phys. Commun. 130 (2000) 260.

[9] S. Jadach, J. Kühn, Z. Was, Comput. Phys. Commun. 64 (1991) 275; S. Jadach, et al., Comput. Phys. Commun. 76 (1993) 361.

[10] S. Jadach, W. Placzek, B.F.L. Ward, Phys. Lett. B 390 (1997) 298.

[11] T. Sjöstrand, et al., Comput. Phys. Commun. 135 (2001) 238; T. Sjöstrand, Comput. Phys. Commun. 82 (1994) 74.
[12] E. Accomando, A. Ballestrero, Comput. Phys. Commun. 99 (1997) 270; E. Accomando, A. Ballestrero, E. Maina, Comput. Phys. Commun. 150 (2003) 166.

[13] F.A. Berends, P.H. Daverveldt, R. Kleiss, Comput. Phys. Commun. 40 (1986) 271

[14] D. Bardin, et al., Comput. Phys. Commun. 133 (2001) 229.

[15] ALEPH Collaboration, DELPHI Collaboration, L3 Collaboration, OPAL Collaboration, SLD Collaboration, LEP Electroweak Working Group, SLD Electroweak and Heavy Flavour Groups, Phys. Rep. C 427 (2006) 257.

[16] A. Djouadi, et al., Z. Phys. C 56 (1992) 289. 